EDutzc EDUEC. Revista Electrónica de Tecnología Educativa.

Número 64 / Junio 2018

\title{
DISEÑO Y EVALUACIÓN DE UN REPOSITORIO ABIERTO DE RECURSOS DIDÁCTICOS PARA LA ENSEÑANZA DE LA HISTORIA RECIENTE
}

\author{
DESIGN AND ASSESSMENT OF AN OPEN REPOSITORY OF EDUCATIONAL \\ RESOURCES FOR THE TEACHING OF RECENT HISTORY \\ Diego Miguel-Revilla; dmigrev@sdcs.uva.es \\ María Sánchez-Agustí; almagosa@sdcs.uva.es \\ Jesús María Moro-Bengoechea; jesusmaria.moro@uva.es \\ Universidad de Valladolid
}

\section{RESUMEN:}

El presente estudio describe el proceso de creación de un repositorio abierto de recursos didácticos ligados a la enseñanza de la Historia reciente, y su evaluación a través de una intervención con 86 alumnos de cuarto curso de la ESO en dos ciudades. Se detalla la integración de los materiales en un entorno digital, examinando su utilidad a través de un análisis cualitativo. Los resultados indican que el trabajo directo del alumnado con estas fuentes históricas facilita la comprensión de la materia, resultando especialmente motivadores los recursos audiovisuales menos usuales (canciones, concursos y viñetas). Se discute sobre la necesidad de favorecer la difusión y colaboración docente mediante un uso adecuado de los repositorios.

Palabras clave: recursos educativos abiertos, repositorio educativo, TIC, enseñanza de la Historia, tecnología educativa

\section{ABSTRACT:}

This paper describes the creation process of an open repository of educational resources linked to the teaching of recent history, and its assessment using an intervention with 86 fourth-year secondary education students in two different cities. The integration of the resources is described, examining their utility by using a qualitative analysis. Results show that students' direct work with these historical sources eases history comprehension, resulting especially motivational those audiovisual resources that are least frequently used (songs, contests and cartoons). The necessity of potentiating the diffusion and educational collaboration by an adequate use of repositories is discussed.

Keywords: open educational resources, educational repository, ICT, history education, educational technology. 


\section{INTRODUCCIÓN}

La democratización del acceso a las tecnologías para la información y la comunicación (TICS) ha proporcionado oportunidades muy diversas a los docentes, no solamente en relación con la potencial implementación de nuevas metodologías de enseñanza, sino también favoreciendo mayores oportunidades de implicación, tanto por parte de los estudiantes como desde el propio profesorado.

Hoy en día, la utilización de herramientas digitales puede observarse de forma habitual en una gran mayoría de los centros educativos, donde las clases basadas en el uso de presentaciones multimedia o de recursos audiovisuales se han convertido en algo común, e incluso en ocasiones, rutinario, sin que por ello se hayan transformado significativamente los métodos de trabajo (Area-Moreira, 2010). La adecuada implementación de las tecnologías en las aulas, pese a las dificultades que ello conlleva, se ha convertido, por tanto, en uno de los objetivos primordiales de los procesos de innovación educativa, mediante los que se procura que el uso de la tecnología no se limite a una mera presencia en los contextos formativos, sino que su utilización tenga un sentido pedagógico coherente (Mishra \& Koehler, 2006; Richards, 2006), adecuando las prácticas docentes a la nueva realidad.

De forma concreta, el interés de la investigación descrita en este artículo se relaciona con la enseñanza y el aprendizaje de la Historia. Ambos son procesos complejos, muy dependientes de los diferentes contextos escolares en los que se llevan a cabo, pero que pueden ser potenciados mediante la integración de las tecnologías (Haydn, 2011).

\section{ESTADO DE LA CUESTIÓN}

Una de las características propias de la disciplina de la Historia es la existencia de multitud de fuentes capaces de convertirse en recursos didácticos de gran utilidad para su enseñanza. Los recientes avances en su proceso de digitalización han permitido facilitar su difusión, favoreciendo la posibilidad de que, como indica Barton (2005), los docentes puedan aprovecharlas para trabajar en torno a la evidencia, con el objetivo de fomentar la curiosidad, ayudar a una mejor comprensión de la perspectiva de los agentes del pasado, y de potenciar la realización de trabajos de investigación en el aula.

Entendiendo la facilitación del acceso a estos materiales como una tarea esencial y propia de la colaboración educativa (McGreal, Kinuthia, \& Marshall, 2013), y a pesar del surgimiento gradual de repositorios digitales, las investigaciones reflejan que los docentes españoles utilizan estas herramientas de forma escasa (Sigalés, Mominó, Meneses, \& Badia, 2008). Aunque los datos más recientes indican que menos de la mitad de los docentes hacen uso de repositorios educativos, las cifras parecen haber aumentado comparativamente en los últimos 
años (Gómez Gómez et al., 2017). Conviene tener en cuenta estudios como el llevado a cabo por Lee, Doolittle y Hicks (2006) en el contexto estadounidense, donde advierten acerca de la tendencia del profesorado (en este caso concreto, de Historia y Ciencias Sociales) a usar páginas web generalistas en lugar de repositorios o archivos especializados a la hora de buscar y aplicar recursos didácticos.

Estos repositorios adquieren mayor relevancia cuando adoptan un carácter libre, alojando recursos educativos abiertos (OERs), entendidos como aquellos "materiales educativos digitalizados y herramientas ofrecidas libremente para ser usadas para enseñar, aprender e investigar" (Del-Valle-Jiménez, Celaya-Ramírez, \& Ramírez-Montoya, 2016, p. 152). Éstos asumen cuatro características esenciales: su capacidad para ser reutilizados, revisados, reconfigurados y redistribuidos (Kumar, 2017), incentivando, mediante su integración y categorización en plataformas específicas, el poder ser compartidos entre los diferentes actores de la comunidad educativa.

Pese a la existencia de múltiples experiencias ligadas al uso de la tecnología para educación histórica, éstas suelen limitarse a la utilización de recursos digitalizados, en ocasiones integrados en blogs, wikis o redes sociales (Haydn, 2013; Rivero \& Mur, 2015). Aunque el trabajo en torno a las fuentes históricas y su recopilación en archivos o repositorios se valora como positivo para fomentar la comprensión de la naturaleza fragmentaria de la disciplina y fomentar la colaboración docente (Hangen, 2015; Tally \& Goldenberg, 2005), las experiencias prácticas son extremadamente escasas (Green, Ponder, \& Donovan, 2014).

Ante el reto de utilizar los marcos más adecuados para la creación, selección y difusión del trabajo centrado en la enseñanza de la Historia reciente, este repositorio ha sido puesto en pie como un producto del Proyecto HISREDUC (EDU2013-43782-P) (Sánchez-Agustí, González-Gallego, Miguel-Revilla, \& MartínezRodríguez, 2015), integrando recursos de forma efectiva en un entorno digital de aprendizaje, cuyo análisis centra la atención del presente estudio.

\section{DISEÑO DE LA INVESTIGACIÓN}

\subsection{Intereses y objetivos de la investigación}

Este estudio parte de la relevancia de los repositorios de recursos como elementos centrales para la formación histórica, asumiendo que el diseño y la integración didáctica de dichos materiales digitales en la práctica docente toman un importante valor, condicionando el modo de trabajo en el aula y la recepción de la materia por parte del alumnado, pero también del profesorado (Crowe, 2004).

Entendiendo, por tanto, la importancia de contar con un espacio digital centralizado que facilite la labor de los docentes a la hora de seleccionar, adaptar 
y compartir recursos de cariz histórico, se establece como primer objetivo el desarrollo e implementación de un repositorio de recursos didácticos para la enseñanza de diferentes procesos propios de la Historia reciente, seleccionados en el marco del Proyecto HISREDUC.

Como segundo objetivo, se busca analizar la eficacia de estos recursos para facilitar el aprendizaje de la Historia, implicar y atraer el interés del alumnado de cuarto curso de la ESO, tras una intervención realizada en torno a la enseñanza de la Transición española en dos centros educativos, donde se produjo una integración del repositorio en un entorno digital de aprendizaje basado en Moodle.

\subsection{Contexto y participantes}

El repositorio de recursos diseñado y utilizado en el presente estudio (repositorio.historiarecienteenlaeducacion.com) comenzó su construcción en el año 2015, momento de inicio del Proyecto HISREDUC, y ha continuado siendo actualizado de forma periódica hasta la actualidad, con la intención de ampliar sus contenidos, añadiendo nuevas características, secciones y recursos. El entorno digital de aprendizaje (historiarecienteenlaeducacion.com) ha sido diseñado en paralelo, favoreciendo el proceso de integración de los recursos.

La intervención con alumnos de Educación Secundaria se llevó a cabo en los meses de mayo y junio de 2016. En la misma, se trabajó con un total de 86 estudiantes (46 alumnos y 40 alumnas) de cuarto curso de la ESO, pertenecientes a dos Institutos de Educación Secundaria (IES). En el primero de los centros, situado en la ciudad de Burgos, se trabajó con un solo grupo, mientras que en el segundo, situado en una localidad vallisoletana, se pudo contar con dos. En ambos casos, las intervenciones se orientaron en torno a la enseñanza del proceso de transición a la democracia en España, en el marco de la asignatura Geografía e Historia.

La intervención se llevó a cabo durante un total de seis sesiones distribuidas en tres semanas. En cinco de estas sesiones, los alumnos trabajaron con ordenadores de sobremesa en las salas de informática de los centros, accediendo a los recursos del repositorio presentes en el entorno digital de aprendizaje, así como en ocasiones puntuales, al propio repositorio. La sexta sesión, de evaluación, sirvió para aplicar el instrumento descrito a continuación, con el fin de obtener información adicional sobre la experiencia.

\subsection{Metodología e instrumentos}

Se ha diseñado una investigación eminentemente cualitativa, al entenderse que para valorar esta experiencia adecuadamente es necesario tener en cuenta el contexto en el que se ha llevado a cabo, así como las observaciones de los participantes y las interpretaciones que de éstas puedan hacerse, centrándose en DOI: https://doi.org/10.21556/edutec.2018.64.981 
el análisis de concepciones emergentes y no necesariamente homogéneas (Stake, 2010).

Partiendo de esta base, el instrumento de recogida de información es un cuestionario en el que preguntas de respuesta abierta, en las que se indaga acerca del papel de los recursos usados en el proceso de aprendizaje y donde se examina la tipología de materiales utilizados, se combina con una valoración general, a través de una pregunta de respuesta cerrada, sobre el grado de interés despertado por el uso de los recursos. Este último aspecto ha sido cuantificado haciendo uso de una escala Likert (1: nada de acuerdo; 5: muy de acuerdo).

El procesamiento de la información se ha llevado a cabo haciendo uso de SPSS para el tratamiento de los datos más descriptivos, y utilizando ATLAS.ti para llevar a cabo la codificación y análisis de las respuestas abiertas. Para esta labor se ha seguido una estrategia basada en la identificación de categorías emergentes (Corbin \& Strauss, 2008) relacionadas con aspectos como la recepción del uso de recursos, la implicación del alumnado, y la facilitación del aprendizaje y comprensión de la materia, capaces de complementar las categorías preestablecidas por el propio diseño del cuestionario, ofreciendo información adicional sobre la intervención.

\section{DISEÑO, IMPLEMENTACIÓN E INTEGRACIÓN DEL REPOSITORIO}

Para la puesta en marcha del repositorio de recursos se ha utilizado Omeka, una plataforma libre y de código abierto diseñada por el Centro Roy Rosenzweig para su aplicación por parte de museos, bibliotecas u otras instituciones, además de en diversos contextos relacionados con la enseñanza de la Historia (Marsh, 2013; Molebash, Lee, \& Friedman, 2013).

El repositorio, instalado en un servidor propio, se estructura con diversos objetivos en mente:

- Recopilar una selección comisariada de recursos sobre cinco temáticas propias de la Historia reciente (ver Tabla 1).

- Proporcionar una plataforma centralizada en la que los docentes puedan encontrar fuentes históricas de diferente naturaleza y destacado potencial didáctico para aplicar en sus clases.

- Facilitar la difusión y modificación de los materiales seleccionados para su adaptación a cada uno de los contextos escolares. La naturaleza del repositorio, de código abierto, y con capacidad para la implementación de comentarios y de un sistema para el envío externo de materiales, asume los indicadores de calidad descritos por Atenas y Havemann (2014).

Junto al sistema de etiquetas, la adscripción de Omeka a la Dublin Core Metadata Initiative (DCMI) favorece la estandarización de las categorías de metadatos, 
abriendo la posibilidad de transmitir o migrar la información entre plataformas alternativas.

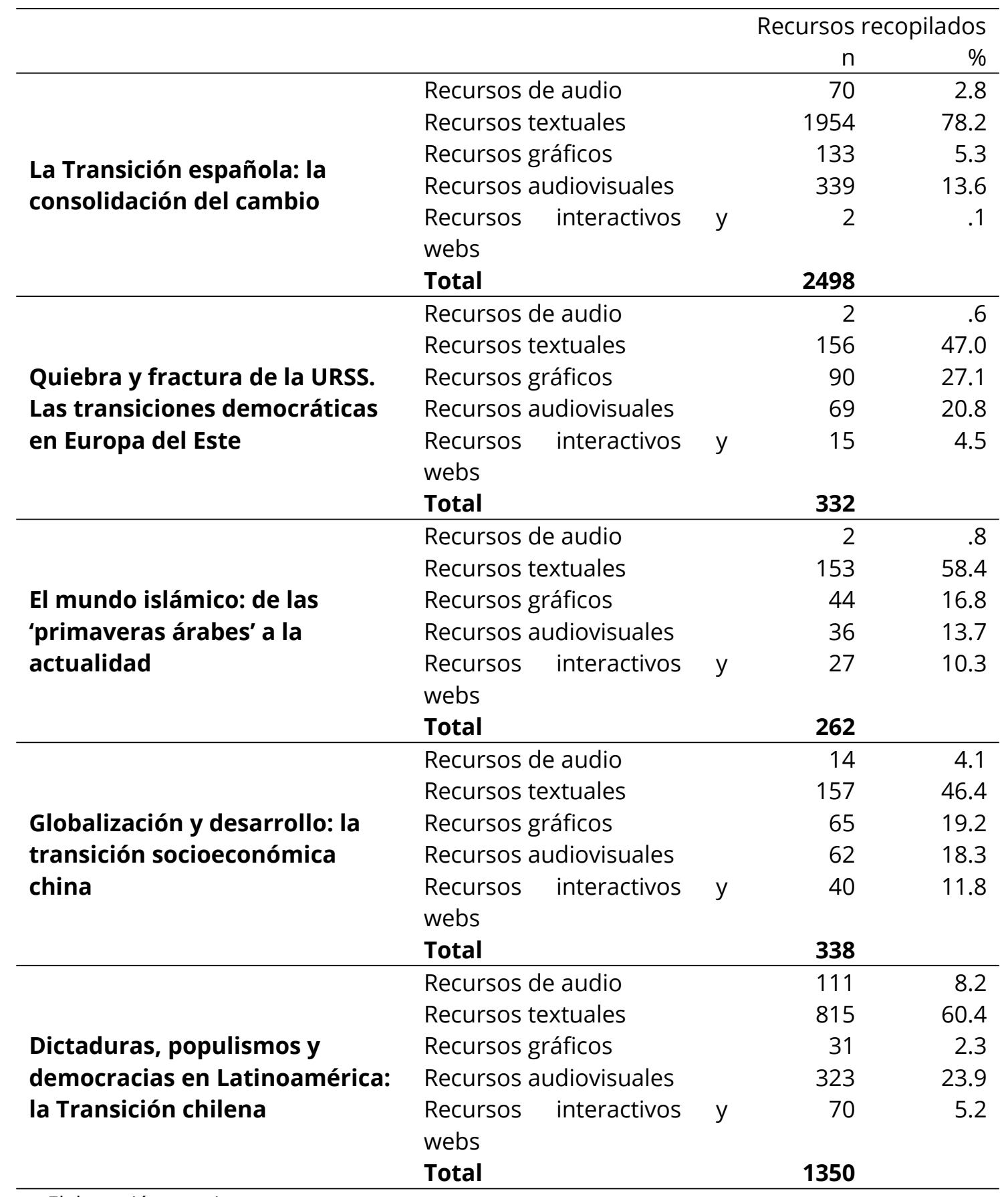

Fuente: Elaboración propia.

Tabla 1: Distribución de los recursos del Repositorio HISREDUC por tipo y temática

Esta recopilación tiene su origen en las investigaciones llevadas a cabo en el marco del Proyecto TRADDEC (EDU2009-09775), centradas en los procesos de transición a la democracia en España y Chile. Partiendo de los materiales obtenidos durante esta etapa, el Repositorio HISREDUC ha sido ampliado para alojar recursos de las cinco temáticas citadas con anterioridad. Actualmente, el 
repositorio se nutre de un total de 4780 recursos clasificados según categorías y etiquetas, como puede verse en la Figura 1.

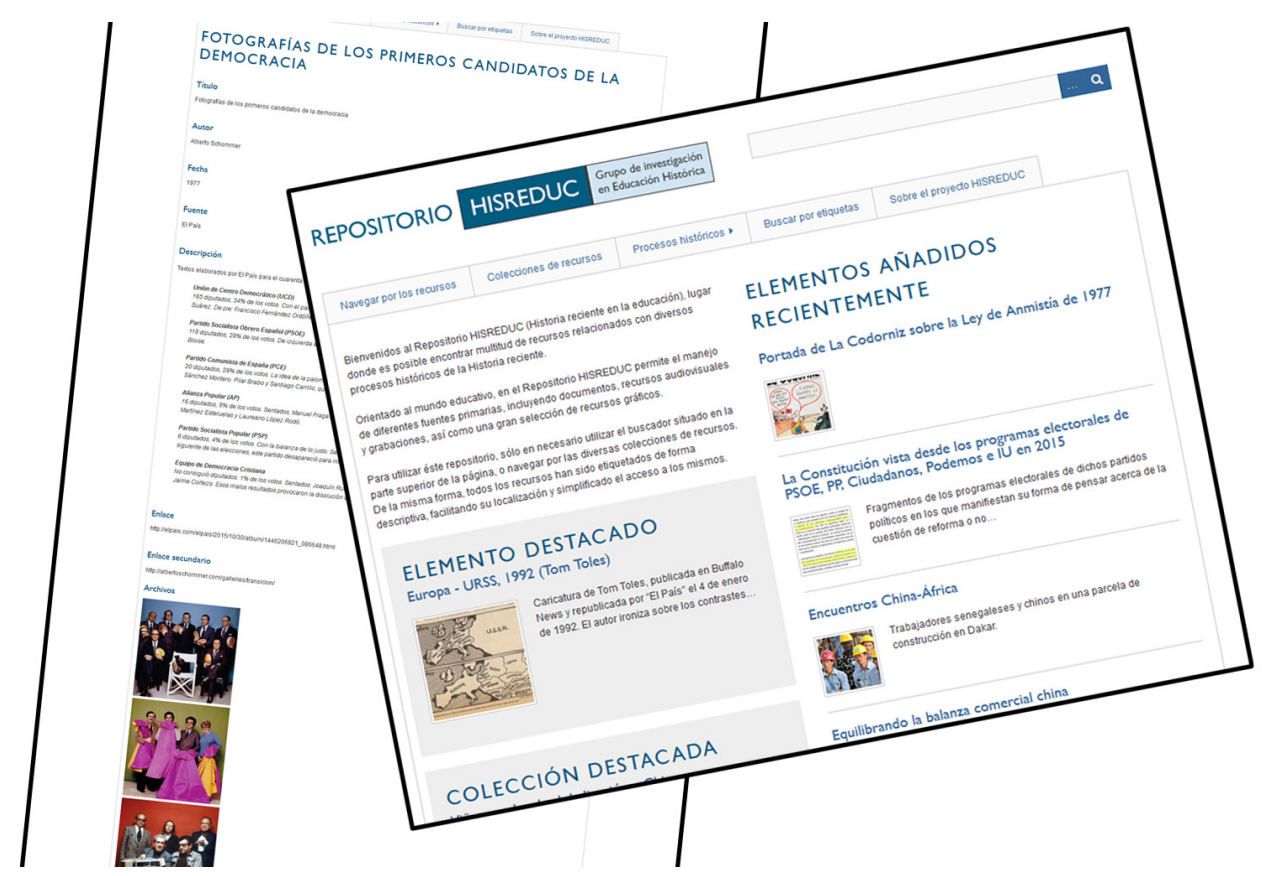

Figura 1. Portada del Repositorio HISREDUC y ficha de uno de sus recursos.

Pese a la disponibilidad y el acceso abierto y público del repositorio, la presente investigación detalla la implementación de recursos específicos en el entorno digital de aprendizaje (historiarecienteenlaeducacion.com) usado para la intervención en los centros de Educación Secundaria. Los materiales se mantuvieron alojados en el repositorio, para ser luego integrados mediante código HTML en las diferentes secciones del entorno digital, como puede observarse en la Figura 2. En esta ocasión, únicamente fue utilizada una selección de fuentes ligadas con la Transición española, al ser ésta la temática a trabajar con el alumnado de cuarto curso de la ESO. Entre los recursos destaca el uso de videoclips de canciones de la época, de fragmentos de documentales y de fotografías, así como de portadas de periódico y revistas, viñetas, gráficos interactivos o anuncios de televisión.

Durante la experiencia, los estudiantes dispusieron de varias oportunidades para visitar el repositorio de forma directa y conseguir más información sobre las diferentes fuentes históricas utilizadas en el entorno. Este proceso se estructuró de forma guiada, bajo control del docente, encargado de decidir el tiempo disponible para cada uno de los procesos de aprendizaje autónomo. 


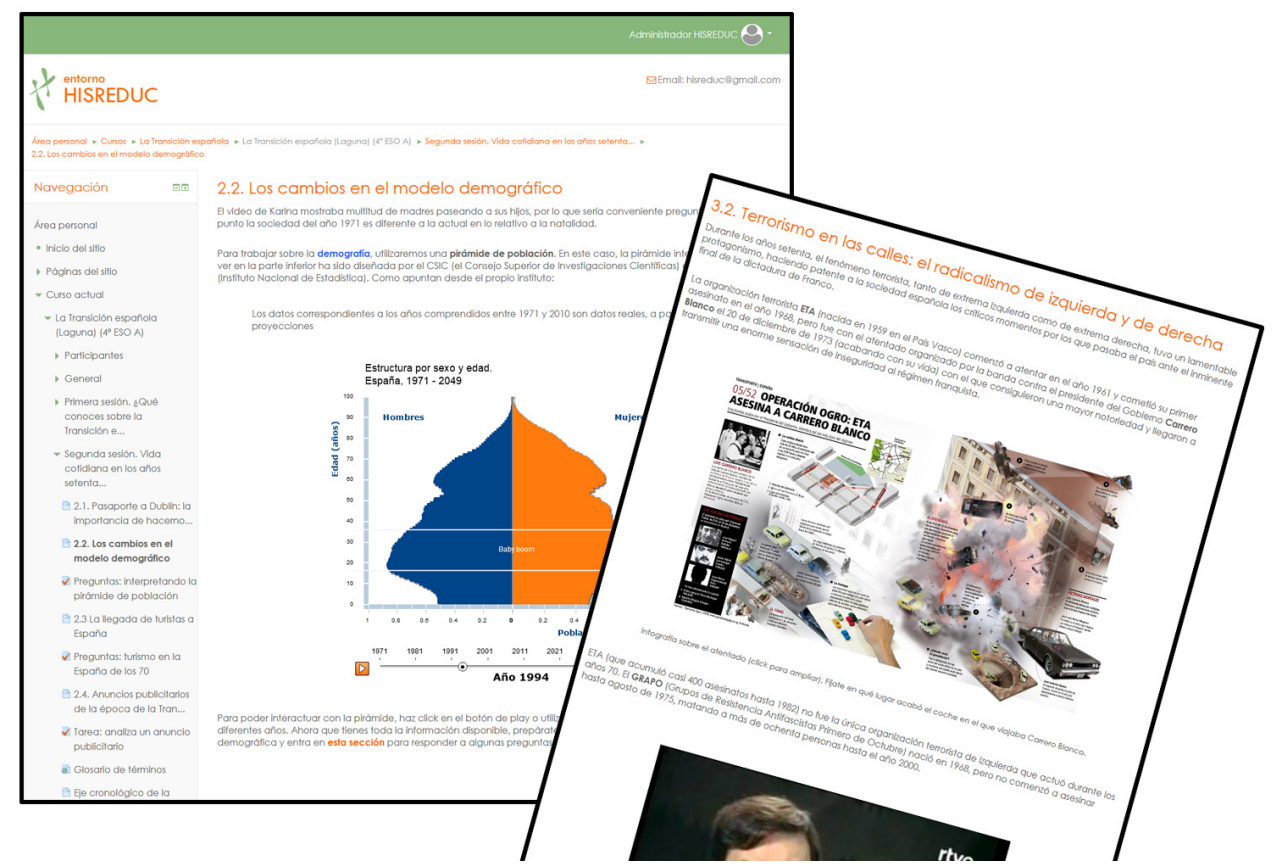

Figura 2. Integración de varios de los recursos didácticos del repositorio en el entorno digital.

\section{ANÁLISIS DE RESULTADOS}

Tras la intervención, y utilizando una jornada adicional, los participantes fueron preguntados acerca de su experiencia a lo largo de las sesiones. En primer lugar, con el objetivo de valorar su opinión global acerca del uso de las diferentes fuentes históricas presentes en el repositorio e integradas en el entorno, se les pidió que calificaran de 1 a 5 el interés despertado por los recursos, obteniéndose respuestas por parte de 78 de los 86 participantes

Los resultados, indicados en la Tabla 2, indican un alto grado de satisfacción con los recursos utilizados, lo que ha sido analizado en profundidad en secciones posteriores. Es de interés recalcar que las diferencias encontradas entre el grupo 1 (centro burgalés) y los grupos 2 y 3 (centro vallisoletano) pueden deberse principalmente al método de trabajo utilizado de forma habitual por el docente a cargo del primer grupo, más ligada a un uso motivador de las tecnologías.

\begin{tabular}{|c|c|c|c|c|c|c|c|c|}
\hline \multicolumn{3}{|c|}{ Grupo 1} & \multicolumn{3}{|c|}{ Grupo 2} & \multicolumn{3}{|c|}{ Grupo 3} \\
\hline$M$ & SD & $\mathrm{n}$ & $M$ & SD & $\mathrm{n}$ & $M$ & SD & $\mathrm{n}$ \\
\hline 3.97 & .89 & 32 & 4.45 & .67 & 22 & 4.29 & .96 & 24 \\
\hline
\end{tabular}

Nota: $\mathrm{M}$ = media; $\mathrm{SD}$ = desviación estándar; $\mathrm{n}$ = número de respuestas. Fuente: Elaboración propia.

Tabla 2: Interés despertado por los recursos utilizados en la intervención

\subsection{Los recursos como facilitadores del aprendizaje histórico}

El examen de las respuestas abiertas de los participantes corrobora una visión positiva acerca de los recursos utilizados durante la intervención. Esta percepción es muy clara para 55 de los encuestados, quienes argumentan que "es una manera muy buena de aprender Historia" (37, G2), o que "es una forma original de 
enseñar aspectos de la Historia" $(65$, G3). Esta visión positiva es algo más matizada para tres estudiantes, para quienes estas fuentes "son algo interesantes, pero tampoco mucho" (41, G2), en palabras de uno de ellos. Por otro lado, solamente dos alumnos muestran una visión negativa, algo ligado a la dificultad de interpretación en, al menos, uno de los casos, ya que al encuestado no le parecieron interesantes "porque no las he entendido" $(4, \mathrm{G} 1)$.

Las razones explicitadas por los alumnos a la hora de indicar su interés por el uso de las diferentes fuentes históricas incluyen la adecuada selección realizada, un aspecto citado por tres de los participantes. Para éstos, los diferentes recursos "estaban bien elegidos para entender la explicación" (35, G2), "el material utilizado es amplio y está muy bien ya que son ejemplos reales que se utilizaban antaño" (56, G2), indicando también que son recursos "que en papel es difícil tener" (21, G1).

En ocho ocasiones, los recursos didácticos se relacionan con su capacidad para hacer las clases más amenas y dinámicas. Si para uno de los encuestados, la integración de las fuentes del repositorio "nos hace entender mejor de una forma más entretenida" $(42, \mathrm{G} 2)$, otro de sus compañeros reitera esta misma idea, destacando un tipo de recurso específico: "Era entretenido y a la vez novedoso escuchar canciones de esta época" $(79, \mathrm{G} 3)$. La palabra entretenido es repetida por siete de estos ocho estudiantes, combinándose con otros términos como divertido y ameno: "Es más entretenido y divertido" (24, G1); "Lo hacía más ameno" (1, G1).

La razón más citada por los encuestados a la hora de defender su interés por los recursos utilizados tiene que ver con su utilidad, sobre todo a la hora de facilitar la comprensión de la materia. Son 21 los alumnos que hacen referencia a este aspecto, ya que, como destaca uno de los participantes, "ayudan a retener conceptos, aprender sobre el tema y son interesantes" (12, G1), y como apunta otro, "con ellos comprendías la Historia [sic] diferente y sirven para recordarlo mejor" (82, G3). Esta opinión es reiterada por muchos de los estudiantes, recalcando la idea de que "con recursos audiovisuales los contenidos pueden asimilarse mejor" (64, G3), pues, en palabras de otro de los encuestados, "me parece mejor que los libros de texto porque es todo más visual y queda más claro" (71, G3).

La ayuda de las fuentes históricas para acercarse al contexto del momento es un aspecto señalado por varios de los participantes, quienes argumentan que "se trabajó con videos, fotos, periódicos de la época y eso te hace entender mejor lo que sucedió" (42, G2). A la vez, se liga con una mayor facilidad para comprender la mentalidad del momento, pues "las canciones, viñetas... etc [sic] nos dan una idea de la sociedad más cercana" (47, G2), algo que también se percibe como útil para comprender la relevancia de la cultura popular a la hora de examinar el pasado, pues tal y como defiende un alumno, "te das cuenta de cómo tienen relación canciones conocidas con el periodo de Transición" (8, G1). 


\subsection{Selección y valoración de los recursos}

Como forma de profundizar en las preferencias de los participantes, éstos fueron preguntados acerca de cuáles de los recursos históricos con los que trabajaron percibieron como más interesantes. Tras el análisis de las respuestas, y aunque 25 personas afirman explícitamente que no eliminarían ninguno de ellos, indicando que "todos me gustaron, y no cambiaría nada" (44, G2), que "los dejaría igual" (3, G1), o que "había alguno más aburrido que otro, pero no los cambiaría ya que se entienden bien" (27, G1), una gran mayoría de los participantes sí identifican qué fuentes les gustan más.

De forma genérica, los encuestados muestran una preferencia, en primer lugar, por el uso de videoclips de canciones (36 menciones), seguido por la utilización de juegos interactivos (22 referencias), vídeos de distinto tipo (14 ocasiones) y viñetas (7 menciones). Aunque la utilización de fotografías o imágenes, textos u otros elementos también son citados, lo son en un grado mucho menor que los otros tipos de recursos.

De forma particular, la utilización de un vídeo con la cabecera del programa Un, dos, tres... responda otra vez del año 1976, integrado en una actividad gamificadora en la que se utilizó la herramienta online Kahoot, fue citado en 22 ocasiones. La sesión en la que se implementó (la primera) también fue identificada como su preferida por un total de 33 encuestados. La novedad de la experiencia, y el hecho de que fuera éste el primer día de la intervención, han podido afectar a la percepción sobre los materiales usados.

El segundo recurso más apreciado es un videoclip con preguntas interactivas correspondiente a la canción En un mundo nuevo de Karina (1971), unido a otro vídeo del programa de TVE Pasaporte a Dublín, emitido un año antes para elegir al representante español para Eurovisión. Ambos, como conjunto, son citados por ocho participantes, encontrando expresiones como "me ha gustado mucho" (24, G1) de forma muy frecuente.

También son referenciadas, de forma específica, la canción Libertad sin ira (1976), de Jarcha (en seis ocasiones), la canción L'estaca (1968), de Lluís Llach (cuatro veces), y Habla, pueblo, habla (1976), del grupo Vino Tinto (un total de tres menciones). Los participantes citan, además, otros recursos como sus preferidos, como algunos anuncios de publicidad concretos de la época de la Transición (en dos ocasiones), o una pirámide demográfica interactiva (también dos veces).

Aunque también se ofreció la oportunidad de valorar los recursos menos interesantes, frente a un total de 86 referencias relativas a los materiales preferidos, solamente es posible encontrar 38 menciones a los peor valorados, indicando una visión de conjunto positiva. Los recursos del repositorio con peor recepción incluyen algunas de las canciones (ocho menciones), los periódicos digitalizados (cuatro referencias), las viñetas (citadas en dos ocasiones), la 
pirámide de población (dos menciones), y los vídeos de diverso tipo, incluyendo los relacionados con aspectos políticos (dos referencias). Curiosamente, elementos muy bien valorados, como la canción de Karina y el vídeo de Eurovisión fueron nombrados también de forma específica por seis participantes en sentido opuesto, aunque ninguno de ellos llegue a expresar las razones de este rechazo.

\section{DISCUSIÓN Y CONCLUSIONES}

La adecuada utilización de las herramientas digitales para favorecer el proceso de enseñanza-aprendizaje en la educación obligatoria no es una labor simple, debido a la necesidad de conjugar aspectos ligados al uso de las tecnologías con elementos pedagógicos y ligados a los contenidos (Mishra \& Koehler, 2006). En el caso de la enseñanza de la Historia, este proceso puede ayudarse de la utilización de numerosos recursos que, aplicados de una manera apropiada, pueden facilitar la formación de un alumnado crítico y capaz de manejar con criterio la evidencia histórica para entender los procesos del pasado y comprender el mundo que les rodea (Barton, 2005).

El diseño e implementación del repositorio de recursos descrito en esta investigación ha tratado de no limitarse a ser una recopilación de materiales con potencial didáctico, como advierte Haydn (2011), sino también de favorecer el cambio metodológico en una asignatura fuertemente anclada en estrategias de enseñanza muy memorísticas, esperando, además, potenciar el intercambio y la colaboración entre docentes.

Atendiendo a los resultados, la experiencia descrita a lo largo del presente artículo indica una recepción especialmente favorable a la integración de recursos en el aula. Tanto el análisis cualitativo de las opiniones expresadas por los participantes, como la valoración cuantitativa, coinciden en indicar una percepción del alumnado acerca del uso de las fuentes históricas como algo novedoso e interesante. En este caso, es posible corroborar cómo la utilización de recursos digitalizados ha sido capaz de involucrar a los participantes en el proceso de interpretación y análisis de la Historia, algo destacado por Green, Ponder y Donovan (2014) como uno de los rasgos esenciales del uso de archivos o repositorios digitales en las aulas.

La involucración del alumnado puede detectarse en dos vertientes diferenciadas. En primer lugar, es relevante apuntar que el uso de los recursos es valorado mayoritariamente como un elemento clarificador y capaz de favorecer la comprensión en el proceso de aprendizaje de la Historia. Desde este punto de vista, destaca el hecho de que los participantes hayan considerado los recursos utilizados como útiles para percibir la perspectiva de los protagonistas históricos y, por tanto, para acercarse con mayor facilidad al pasado. Este último aspecto, esencial en el desarrollo de un trabajo más disciplinar, parece verse favorecido 
por la naturaleza de las fuentes, identificadas como cercanas y capaces de despertar el interés del alumnado.

En segundo lugar, y precisamente en relación con la implicación de los participantes, es destacable que, pese a que el uso de fuentes digitalizadas no haya sido identificado exclusivamente como un aspecto entretenido o motivador, el análisis realizado acerca de los recursos mejor y peor recibidos indica que los elementos audiovisuales (como los videoclips de canciones) e interactivos fueron especialmente bien acogidos. En esta ocasión los resultados encontrados por Tally y Goldenberg (2005) parecen encontrar aquí una cierta réplica, al percibirse que el uso de las fuentes digitalizadas ha despertado un mayor interés en los participantes, algo que a su vez parece haber implicado una visión más benévola de la disciplina, favoreciendo además un tipo de trabajo en el que las fuentes asumen un protagonismo esencial para una comprensión en profundidad de la Historia.

A modo de conclusión, y pese a los retos que todavía quedan por solventar en torno al uso generalizado de herramientas colaborativas, incluidos archivos y repositorios, en el marco de la educación histórica (Hangen, 2015), no cabe duda de la relevancia de la puesta en marcha de medidas con la finalidad de favorecer la reutilización de materiales y la implicación de la comunidad educativa (McGreal et al., 2013). Desde este punto de vista, conviene recordar la necesidad de establecer criterios de calidad, como los destacados por Atenas y Havermann (2014), potenciando repositorios más abiertos, pero también más participativos. Queda pendiente, como labor orientada a futuras actuaciones, no solamente la ampliación de los materiales recopilados, sino también un mayor esfuerzo en torno a su visibilización para el fomento de una mayor facilidad de difusión, reestructuración e integración en las aulas.

\section{REFERENCIAS}

Area-Moreira, M. (2010). El proceso de integración y uso pedagógico de las TIC en los centros educativos. Un estudio de casos. Revista de Educación, 352, 77-97. Recuperado de http://www.revistaeducacion.educacion.es/re352_04.html

Atenas, J., \& Havemann, L. (2014). Questions of Quality in Repositories of Open Educational Resources: A Literature Review. Research in Learning Technology, 22(October). https://doi.org/10.3402/rlt.v22.20889

Barton, K. C. (2005). Primary Sources in History: Breaking Through the Myths. Phi Delta Kappan, 86(10), 745-753. https://doi.org/10.2307/491903

Corbin, J., \& Strauss, A. (2008). Basics of Qualitative Research: Techniques and Procedures for Developing Grounded Theory ( $3^{\mathrm{a}}$ ed.). Thousand Oaks: SAGE Publications.

Crowe, A. R. (2004). Teaching by Example: Integrating Technology into Social Studies Education Courses. Journal of Computing in Teacher Education, 20(4), 159-165. 
Recuperado

de

http://www.tandfonline.com/doi/abs/10.1080/10402454.2004.10784500

Del-Valle-Jiménez, D., Celaya-Ramírez, R., \& Ramírez-Montoya, M. S. (2016). Apropiación tecnológica en el movimiento educativo abierto: Un estudio de casos de prácticas educativas abiertas. Revista Iberoamericana de Educación, 70(1), 149-166. Recuperado de http://rieoei.org/rie_contenedor.php?numero=7186

Gómez Gómez, M., Martínez Domínguez, L. M., Paz-Albo Prieto, J., Bailén, E., Cordero, C., Largo Burgos, J., ... Calvo, J. (2017). III Estudio sobre el uso de la tecnología en el aula. Informe de resultados. Madrid: Blink Learning.

Green, T., Ponder, J., \& Donovan, L. (2014). Educational Technology in Social Studies Education. En J. M. Spector, M. D. Merrill, J. Elen, \& M. J. Bishop (Eds.), Handbook of Research on Educational Communications and Technology (4 ${ }^{\mathrm{a}}$ ed., pp. 573-582). London: Springer.

Hangen, T. (2015). Historical Digital Literacy, One Classroom at a Time. Journal of American History, 101(4), 1192-1203. https://doi.org/10.1093/jahist/jav062

Haydn, T. (2011). History Teaching and ICT. En I. Davies (Ed.), Debates in History Teaching (pp. 236-248). London: Routledge.

Haydn, T. (Ed.). (2013). Using New Technologies to Enhance Teaching and Learning in History. London: Routledge.

Kumar, M. S. V. (2017). The New Landscape for the Innovative Transformation of Education. Social Research: An International Quarterly, 79(3), 619-631. Recuperado de https://muse.jhu.edu/article/528185

Lee, J. K., Doolittle, P. E., \& Hicks, D. (2006). Social Studies and History Teachers' Uses of Non-Digital and Digital Historical Resources. Social Studies Research and Practice, 1(3), 291-311. https://doi.org/10.1080/00933104.2004.10473253

Marsh, A. C. (2013). Omeka in the Classroom: The Challenges of Teaching Material Culture in a Digital World. Literary and Linguistic Computing, 28(2), 279-282. https://doi.org/10.1093/llc/fqs068

McGreal, R., Kinuthia, W., \& Marshall, S. (Eds.). (2013). Open Educational Resources: Innovation, Research and Practice. Vancouver: UNESCO, Commonwealth of Learning \& Athabasca University. Recuperado de http://oasis.col.org/handle/11599/486

Mishra, P., \& Koehler, M. J. (2006). Technological Pedagogical Content Knowledge: A Framework for Teacher Knowledge. Teachers College Record, 108(6), 1017-1054. https://doi.org/10.1111/j.1467-9620.2006.00684.x

Molebash, P. E., Lee, J. K., \& Friedman, A. M. (2013). The Lincoln Telegrams Project: A Design-Based Research Approach to Simplifying Digital History. Journal of the Research Center for Educational Technology (RCET), 9(1), 50-73. Recuperado de http://www.rcetj.org/index.php/rcetj/article/viewArticle/193

Richards, C. (2006). Towards an Integrated Framework for Designing Effective ICTSupported Learning Environments: The Challenge to Better Link Technology and 
Pedagogy. Technology, Pedagogy and Education, 15(2), 239-255. https://doi.org/10.1080/14759390600769771

Rivero, P., \& Mur, L. (2015). Aprender ciencias sociales en la web 2.0. Íber. Didáctica de las Ciencias Sociales, Geografía e Historia, (80), 30-37.

Sánchez-Agustí, M., González-Gallego, I., Miguel-Revilla, D., \& Martínez-Rodríguez, R. (2015). Entornos de aprendizaje digital para la enseñanza de la Historia reciente en España y Chile. Presentación de un Proyecto de Intervención en Secundaria. Andamio. Revista de Didáctica de la Historia, 2(1), 31-48.

Sigalés, C., Mominó, J. M., Meneses, J., \& Badia, A. (2008). La integración de internet en la educación escolar española: situación actual y perspectivas de futuro. Barcelona: Universitat Oberta de Catalunya.

Stake, R. E. (2010). Qualitative Research. Studying How Things Work. New York: The Guilford Press.

Tally, B., \& Goldenberg, L. B. (2005). Fostering Historical Thinking With Digitized Primary Sources. Journal of Research on Technology in Education, 38(1), 1-21. https://doi.org/10.1080/15391523.2005.10782447

\section{FINANCIACIÓN}

Esta investigación ha sido financiada por el Ministerio de Economía, Industria y Competitividad del Gobierno de España dentro del marco del Proyecto HISREDUC ('La Historia reciente en la educación. Diseño y evaluación de entornos digitales de aprendizaje en la enseñanza secundaria de España y (hile'), con referencia EDU2013-43782-P

\section{Para referenciar este artículo:}

Miguel-Revilla, D., Sánchez-Agustí, M. \& Moro-Bengochea, J.M. (2018). Diseño y evaluación de un repositorio abierto de recursos didácticos para la enseñanza de la Historia reciente. EDUTEC, Revista Electrónica de Tecnología Educativa, 64. Recuperado de: http://dx.doi.org/10.21556/edutec.2018.64.981 